\title{
Avaliação do League of Class: uma plataforma de gamificação estrutural inspirada em League of Legends
}

\author{
Rebeca Veiga Assunção \\ Universidade do Estado do Amazonas \\ Manaus, Amazonas, Brasil \\ rsva.lic@uea.edu.br
}

\author{
Fernanda Pires \\ Universidade do Estado do Amazonas \\ Manaus, Amazonas, Brasil \\ fpires@uea.edu.br
}

\author{
Marcela Pessoa \\ Universidade do Estado do Amazonas \\ Manaus, Amazonas, Brasil \\ msppessoa@uea.edu.br
}

\begin{abstract}
Este artigo trata da construção e validação prototípica da plataforma gamificada denominada League of Class, inspirada no MOBA League of Legends (LoL). A proposta de gamificação permite o cumprimento de missões na submissão de atividades e de desafios em batalha $1 \times 1$ de pergunta-resposta. Na estrutura da construção do sistema foi utilizado o framework 5W2H e princípios do Modelo ARCS de design motivacional. Utilizando a ferramenta Instructional Materials Motivational Scale (IMMS), as avaliações iniciais de sua versão em protótipo (paper prototype), realizada com professores da área de computação, apontaram uma boa recepção ao sistema e suas funções de gerenciamento envolvendo metas, regras e desempenhos.
\end{abstract}

\section{CCS CONCEPTS}

- Gamification $\rightarrow$ Gamified systems; Gamification in education; Designing gamification.

\section{PALAVRAS-CHAVE}

Gamificação, design motivacional, framework 5W2H, ARCS

\section{INTRODUÇÃO}

Gamificação, ou gamification, é definida como a utilização de técnicas, dinâmicas e pensamentos de jogos para tornar as atividades mais divertidas e engajadoras, em contextos diferentes dos jogos [13]. Em contexto educacional pode ser usada para proporcionar motivação e gerar engajamento nos estudantes, com o objetivo de promoção de aprendizagem.

Embora existam expectativas positivas na aplicação da gamificação em ambientes digitais para educação, ainda é insuficiente a disponibilidade/acessibilidade desses sistemas em um cenário nacional; sobretudo como ferramenta complementar às atividades do professor que, por muitas vezes, pode encontrar dificuldades na aplicação de métodos e técnicas, entre outras razões, pela falta de conhecimento ou de ferramentas digitais adequadas a seu contexto [3]. Além disso, muitos estudos empíricos não têm justificado a seleção de elementos específicos [9] que possibilitem, por exemplo, apontar uma arquitetura de "jogo" mais adequada a ser adaptada. Assim como poucos estudos focados no planejamento da gamificação

Fica permitido ao(s) autor(es) ou a terceiros a reprodução ou distribuição, em parte ou no todo, do material extraído dessa obra, de forma verbatim, adaptada ou remixada, bem como a criação ou produção a partir do conteúdo dessa obra, para fins não comerciais, desde que sejam atribuídos os devidos créditos à criação original, sob os termos da licença CC BY-NC 4.0.

EduComp'21, Abril 27-30, 2021, Jataí, Goiás, Brasil (On-line)

(c) 2021 Copyright mantido pelo(s) autor(es). Direitos de publicação licenciados à Sociedade Brasileira de Computação (SBC). envolvem o professor como parte do processo em suas abordagens [22], muitos não analisam um conceito de apoio automatizado ao professor em processos de planejamento [27].

Com ênfase nas etapas da construção de sua gamificação e em avaliações iniciais de sua versão em protótipo (paper prototype), esse artigo apresenta a plataforma de gamificação estrutural intitulada League of Class (LoC), com elementos de jogo inspirados no popular MOBA League of Legends (LoL), inicialmente projetado para atender professores da área de computação, por já terem intimidade com tecnologias. LoC tem como objetivo fornecer ferramentas que possibilitem ao educador gamificar as suas atividades na escola/universidade sem a necessidade de realizar grandes mudanças em seu planejamento. Atribuindo uma identidade de "jogo" às tarefas que o professor passa para suas turmas, tornando possível acompanhar o desenvolvimento dos estudantes a partir de sua evolução em missões e desafios. O desenvolvimento da arquitetura do sistema gamificado foi apoiado pelo framework $5 \mathrm{~W} 2 \mathrm{H}$ e o modelo ARCS (Atenção, Relevância, Confiança e Satisfação) como suporte ao design motivacional.

Este artigo está organizado nos seguintes tópicos: Seção 2 apresenta os trabalhos relacionados; a Seção 3 apresenta os fundamentos teóricos considerados na construção do trabalho; a Seção 4 apresenta o LoC e o planejamento estrutural do sistema; a Sessão 5 apresenta a discussão da análise de avaliações do sistema feitas por professores da área de computação; e a Seção 7 apresenta as considerações finais e trabalhos futuros.

\section{TRABALHOS RELACIONADOS}

A gamificação tem atraído a atenção de pesquisadores com atuação em diferentes níveis de ensino. Neste contexto, o CodePlay [26] é uma plataforma estrutural gamificada baseada em mecânicas de RPG (role-playing games) multiplayer que foi integrada ao sistema juiz on-line CodeBench, como apoio às disciplinas introdutórias de programação, nos cursos superiores de ciências exatas. No ambiente, ao acertar um exercício de programação, é sorteada uma carta ao aluno, fornecendo acesso a lugares bloqueados e a tesouros. Para garantir que haja cooperação entre os alunos, o jogo só avança de fase quanto uma parte dos alunos resolve os exercícios. Nos testes de aplicação da plataforma, não houve diferença significativa no desempenho escolar dos alunos, embora tenha aumentado em $5 \%$ o tempo de permanência do estudantes nas atividades e, em análise mais minuciosa, dobrou o número de estudantes com média 10,0. A pesquisa trouxe sua forte contribuição para a defesa do grande potencial da gamificação de sistemas e, ademais, de sistemas originalmente estruturados com elementos da gamificação, como o proposto neste projeto. 
Com referência ao nome do jogo Call of Duty, a plataforma Call of Code $[11,12]$ foi desenvolvida com o objetivo de incentivar o trabalho colaborativo e a resolução de problemas com o pensamento computacional, ambientada na Segunda Guerra Mundial. Em vídeos tematizados, o aluno observa conceitos lógicos computacionais e recebe desafios a serem cumpridos em "campo de batalha". Esses objetivos agregam pontos de experiência para o aluno favorecendo uma evolução de sua patente. Após a verificação de pontuações evoluções de patente em simulações realizadas em laboratório, os pesquisadores registraram que seus resultados foram animadores, e que os elementos da gamificação utilizados (como pontuação e níveis, também utilizados neste projeto) ajudaram a atingir seus objetivos dentro de suas propostas de pesquisa.

Abordando o impacto das tecnologias e jogos digitais causadas no ambiente da sala de aula, Alves et al. [2] desenvolveram um estudo utilizando conceitos da gamificação na aplicação do jogo MOBA Dota 2, como ferramenta de auxílio em matemática, em um modelo de atividade adaptado para a abordagem do desenvolvimento cognitivo matemático. Defendendo o apropriar-se da cultura da nova geração como algo, no mínimo, inteligente a se fazer [2, p. 18], o estudo trouxe uma abordagem pedagógica de conceitos como educação financeira, probabilidade, análise combinatória, análise de gráficos, domínio de tempo, estratégia e trabalho em equipe, trazendo sugestões pedagógicas adaptáveis de acordo com conteúdo ou tempo de aplicação.

Os sistemas digitais gamificados também podem contribuir no entrave contra problemas sociais, como abordado na pesquisa de Nunes et al. [24]. Na procura de suprir a dificuldade na comunicação durante o tratamento e reinserção social de dependentes químicos, foi desenvolvido um sistema chamado ConneCT em que o paciente responde um conjunto simples de perguntas diariamente, na prevenção de recaídas. Para que o ambiente fosse empregado de forma efetiva e com resultados relevantes, técnicas de gamificação foram incorporadas ao sistema na busca de se obter maior quantidade e frequência de respostas. No entanto, se fez necessário o refinamento de estratégias da gamificação para corresponder aos objetivos e necessidades abordadas através do Framework 5W2H, pois, segundo os autores, facilitou o esclarecimento e a organização das etapas de planejamento do ambiente.

Assim como no projeto de Nunes et al.[24], o Framework 5W2H foi escolhido por se tratar de uma abordagem flexível com o objetivo de "guiar o projeto, o desenvolvimento e a avaliação da gamificação centrada no usuário" [17, p. 1]. Acredita-se que esse método poderá implicar na facilitação de uma escolha apropriada de elementos e estratégias gerais da gamificação.

\section{FUNDAMENTAÇÃO TEÓRICA}

Esta seção descreve a fundamentação teórica necessária para o entendimento da proposta, incluindo gamificação, multiplayer online battle arena e framework $5 \mathrm{~W} 2 \mathrm{H}$.

\subsection{Gamificação}

Existem pelo menos dois tipos de gamificação: estrutural e de conteúdo [14]. Na gamificação de conteúdo aplicam-se elementos e pensamentos de jogos para alterar o próprio conteúdo ou a estrutura da informação, através de métodos de game design. Na gamificação estrutural utilizam-se elementos de jogos para conduzir o processo de aprendizagem sem alterações do conteúdo, podendo ser utilizada para estruturar ambientes digitais em que o estudante tenha acesso a conteúdos distribuídos [14].

Para a construção de uma solução gamificada, os elementos dos jogos são como uma caixa de ferramentas, com itens que podem ser combinados de diferentes maneiras, dependendo da finalidade da aplicação. Werbach e Hunter [28] organizam os diferentes tipos de elementos da gamificação mais comuns em três componentes:

(1) Dinâmica: estruturas implícitas que atribuem coerência às experiências (como emoções e progressão);

(2) Mecânica: elementos de jogos que trazem a ação no sistema gamificado (como desafios, recompensas e estado de vitória);

(3) Componentes do jogo: formas específicas de executar a representação dos outros componentes (como avatar, pontos ou placar).

Através de gostos e preferências, os elementos de jogo estão diretamente ligados aos resultados emocionais e comportamentais do jogador [19]. "Através dos mecanismos da gamificação é possível alinhar os interesses dos criadores dos artefatos e objetos com as motivações dos usuários" [7, p. 16]. Para auxiliar na composição de ambientes, pesquisas tem enveredado para a personalização dos elementos de jogos, segundo as preferências do jogador. Uma das tipologias específicas para a gamificação é proposta por Marczewski, denominada User Type Hexad [20], que descreve seis tipos de jogadores, como segue:

(1) Socialisers: motivados por relacionamentos no sistema e ligações sociais;

(2) Free Spirits: motivados pela autonomia, gostam de explorar o sistema e criar coisas;

(3) Achievers: motivados pelo domínio, procuram aprender coisas novas com desafios;

(4) Philanthropists: grupo altruísta, se prestam em doar e ajudar colegas de alguma maneira, sem esperar recompensas;

(5) Players: motivados por recompensas, buscando o melhor para si mesmos;

(6) Disruptors: motivados pela mudança, no desejo de quebrar as regras para modificações no sistema ou encontrar falhas.

Cada tipo de jogador tem sua diferente fonte de motivação, tornando a motivação um ponto essencial na relação gamificação e aprendizagem [1]. No trabalho atual, espera-se abordar as diferenciadas fontes de motivação com determinados elementos do sistema, buscando construir funções ativadoras e estimulantes no comportamento de forma intrínseca (referente desejos internos e movido por razões próprias) e extrínseca (com estímulos movidos por fatores externos) - tipos de motivação.

\subsection{Multiplayer Online Battle Arena}

$O$ gênero conhecido como MOBA (Arena de Batalha Online para Multijogadores, em português) é um subgênero dos jogos do tipo RTS (real-time strategy) que envolve ação, estratégia e RPG [23]. O princípio da modalidade é simples: dois times se enfrentam em um mapa geralmente dividido em 3 caminhos com o objetivo de destruir a base adversária. Tudo começa com a escolha de um personagem individual; cada personagem possui habilidades próprias. No começo do jogo, as equipes se preocupam em arrecadar recursos 
para compra de itens e aprimorar as habilidades de seus personagens; na fase intermediária a procura de recursos continua, mas os jogadores investem em avançar nas rotas e no domínio do mapa, encontrando como defesa adversária torres e criaturas automáticas; no final do jogo, os times se concentram na invasão da base inimiga, que garante a vitória na partida [4].

No ano de 2019, o número mundial de espectadores de campeonatos de jogos eletrônicos foi de 453,8 milhões, e dentre os principais eventos consagrados da categoria estão os dos jogos do tipo MOBA. "Em 2018, apenas a final de League of Legends (mais conhecido por LoL) teve 99,6 milhões de pessoas assistindo" [5]. A construção do grande sucesso de League of Legends se deu por uma sequência de lançamentos de funcionalidades pioneiras no segmento, como personalização de personagens (skins), além do fornecimento gratuito de demos e comunicação com a comunidade. No trabalho atual, os conceitos de personagens individuais e equipes será incorporado ao sistema, assim como a customização do personagem no estilo fantasy, pontos resgatáveis, nível individual e elos de equipe, indicando uma linha evolutiva de acúmulo de pontos.

\subsection{Framework $5 \mathrm{~W} 2 \mathrm{H}$}

O modelo 5W2H é uma ferramenta de planejamento de ações que é utilizada em análise e gestão de projetos, elaboração de planos de negócios e outras disciplinas de gestão [8]. A nomenclatura $5 \mathrm{~W} 2 \mathrm{H}$ tem origem nas primeiras letras de palavras-chave (em inglês) de 7 perguntas essenciais que dão diretrizes ao processo, como um checklist de circunstâncias que devem ser respondidas. Com o objetivo de fornecer um guia para a criação de um sistema de gamificação, Klock, Gasparini e Pimenta [17] propuseram um framework baseado no modelo, com foco no usuário. De acordo com o modelo, o framework é composto por sete dimensões de análises, ilustradas na Figura 1 e descritas a seguir:

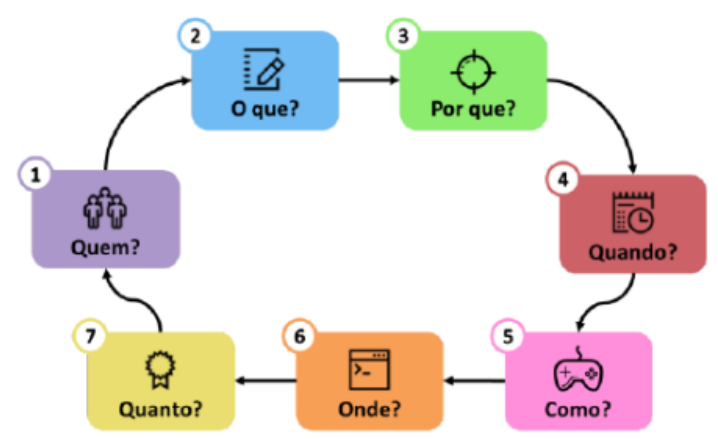

Figure 1: Dimensões do framework 5W2H. Fonte: Klock, Gasparini e Pimenta [17]

(1) "Quem?": identificar o público-alvo e características do usuário como aspectos gerais que influenciam nas experiências (idade, sexo, tipos de personalidade, cultura e preferências em jogos);

(2) "O que?": identificar os comportamentos e tarefas a serem realizadas durante a interação com o sistema para alcançar o propósito da gamificação;
(3) "Por quê?": identificar estímulos a serem despertados para persuasão no realizar dos comportamentos desejados;

(4) "Quando?": identificar situações para os estímulos, envolvendo a evolução do usuário em relação às tarefas e a aplicação de reforços para manter a motivação do usuário;

(5) "Como?": definir o projeto da gamificação, de acordo com as características do usuário e com as dimensões anteriores;

(6) "Onde?": início do processo de implementação do sistema, variando de acordo com o modelo de desenvolvimento;

(7) “Quanto?": avaliar o quanto a implementação da gamificação despertou no público-alvo os estímulos desejados.

Embora considerado como uma das mais completas estruturas referente ao design de gamificação, lhe falta de detalhes sobre a construção de um ambiente motivacional [6]. Na construção do trabalho atual utilizando o framework, buscou-se suprir a carência de um design motivacional incorporando ao processo o modelo ARCS, descrito na próxima sessão.

\subsection{Modelo ARCS como design motivacional}

Para auxiliar na identificação e resolução de problemas motivacionais relacionados ao ensino-aprendizagem em contextos específicos, Keller $[15,16]$ desenvolveu um modelo de design motivacional intitulado ARCS, um acrônimo em que:

(1) Atenção: diz respeito à abordagens para despertar curiosidade, como imagens e ilustrações, interessantes ao públicoalvo;

(2) Relevância: trata-se da importância do objeto tratado em conexão com os objetivos do estudante e como demonstrar a notabilidade ao oferecer o direito de escolha em métodos para aprender;

(3) Confiança: trata do controle pessoal do aluno, da expectativa de sucesso e atribuições de realizações relacionadas às habilidades e esforços;

(4) Satisfação: diz respeito à procura de equilíbrio entre recompensas e reconhecimentos em relação ao esforço produzido.

A proposta é dar diretivas para tornar a aprendizagem intrinsecamente interessante e despertar o interesse em aprender [15]. No desenho da motivação na aprendizagem assistida por tecnologia, o modelo se mostra válido quando é combinado com um processo sistemático de desenvolvimento de projeto [16], assim como se pretende no trabalho atual, considerando os princípios do modelo. O autor defende, ainda, que a motivação é promovida e mantida quando os estudantes executam estratégias auto-reguladoras para atingir uma meta, persistindo em esforços.

\section{PROJETO LEAGUE OF CLASS}

O League of Class é uma plataforma gamificada que tem como objetivo auxiliar o professor na gerência de atividades e motivar os alunos em submissões de tarefas. Com um gerenciamento de pontuações baseadas em pontos atribuídos por submissões de respostas e por bônus comportamentais atribuídos ao aluno, o sistema possibilita o controle dos desempenhos pelo professor.

Na plataforma o professor cria e administra: turmas; equipes de alunos; atividades; desafios (fase do chefão); pontuação bônus e penalidades baseadas no comportamento do aluno; objetivos e 
metas de pontuação. Por sua vez, o aluno: realiza submissões de tarefas; cumpre desafios em forma de quiz-combate aluno vs monstro (fase do chefão); comenta atividades; ganha pontos de experiência e pontos resgatáveis; e realiza a compra de bens virtuais. O mesmo planejamento que o professor realiza com suas avaliações por envio de tarefas podem ser aproveitadas na utilização do sistema. Esperase que o aluno encontre elementos engajadores no sistema, de forma que o proporcione momentos agradáveis de interação e satisfação na submissão de tarefas.

Para ajudar na imersão, o contexto do League of Class é em um lugar chamado Terra do Amanhecer, onde grandes heróis são convocados para seguirem em uma missão de explorar terras desconhecidas. Divididos em equipes, os heróis coletam recursos e experiências, mas sempre em preparação constante, pois, a qualquer momento o terrível Chefão pode surgir, desafiando os heróis para uma batalha. Guerreiros, preparem-se! É uma batalha individual!

A seguir, é apresentado o planejamento da gamificação e sua modelagem, utilizando o modelo ARCS de design motivacional e o framework 5W2H na construção estrutural.

\subsection{Construção e planejamento da gamificação}

Na dimensão "Quem?" foram identificados o público-alvo e as características dos usuários. Embora o sistema considere o professor um usuário, os estudantes serão adotados como público-alvo, com margem de idade acima dos 11 anos, especialmente alunos de disciplinas de informática, pois, além de estarem inseridos em conceitos sobre tecnologia, os jogos do estilo MOBA têm grande aderência entre jovens e adolescentes [23]. Foi adotado como referência cultural o MOBA League of Legends (LoL). Estão sendo considerados os aspectos gerais que influenciam nas preferências e perfis motivacionais (como idade, tipos de personalidade e cultura), e condições de aprendizado relacionados à estrutura do pensamento, de acordo com o estágio de desenvolvimento cognitivo. É importante esse olhar para uma reflexão quanto a Arquitetura da Informação no sistema [18].

Na dimensão “O Que?" são determinados os comportamentos desejados pelo público-alvo ao interagir com o sistema, para alcançar os propósitos da gamificação. A proposta atual possui três objetivos base, fundamentados no modelo ARCS: (1) desempenho, (2) confiança e (3) autonomia. São adotados, também, o objetivo (4) comunicação, para incentivar a comunicação social com os colegas e professor.

Na dimensão "Por quê?" são identificados os estímulos a serem gerados no usuário ("Quem?") para a realização do comportamento desejado ("O que?") e execução de tarefas. Aqui o intuito da ferramenta é abordado, sendo relacionado diretamente com os objetivos de motivação e engajamento. De acordo com o modelo ARCS, é necessário que se conquiste a atenção do aprendiz para que ele tenha interesse na aprendizagem, e isso pode ser feito através do despertar da curiosidade, elementos gráficos e mudanças de ritmos de atividade [16]. Também é possível cativar o aprendiz com a promoção de experiências agradáveis e engajadoras, formalizando os estímulos base desejados em (1) atenção, (2) aprendizagem, (3) relevância e (4) satisfação.
Na dimensão "Quando?", são definidas as situações para o estímulo ("Por Quê?") do usuário (“Quem?") no realizar do comportamento desejado ("O que?"), as tarefas e funções do sistema estão representadas pela Figura 2. A dimensão considera a jornada do jogador e frequências de estímulos:

(1) No sistema, o estudante submeterá respostas em missões como atividades sequenciais, sendo desbloqueadas gradativamente, de acordo com a visualização ou aprovação de resposta das atividades. Assim que a submissão for aceita, o estudante receberá pontos resgatáveis e pontos de experiências.

(2) Os pontos regatáveis poderão ser utilizados para manter o seu perfil de jogador na compra de itens para seu avatar.

(3) O aluno também poderá comentar a atividade disponível, criando um mini-fórum em cada atividade da missão.

(4) Cada aluno terá um nível individual de acordo com o acúmulo de pontos de experiências adquiridas (xpAluno) em missões $(M)$ e em desafios $(D)$ ( $t$ = quantidade de tarefas respondidas, $d=$ quantidade de desafios cumpridos). A Equação 1 apresenta a fórmula usada para calcular a experiência do jogador. O aluno também será integrante de uma equipe, na qual acumulará os pontos de experiência de seus participantes. A cada quantidade de pontos acumulados $(\rho)$, a equipe subirá de nível, adotados como elos de equipe $(e=$ quantidade de estudantes na equipe), calculada conforme Equação 2 .

$$
\begin{gathered}
\text { xpAluno }=\sum_{k=0}^{t} M_{k}+\sum_{z=0}^{d} D_{l} \\
\rho=\frac{\sum_{x=1}^{e} x p \text { Aluno }}{e}
\end{gathered}
$$

(5) O aluno poderá participar de desafios em forma de quizcombate aluno vs monstro, chamada fase do Chefão. A cada rodada o aluno responde uma questão-problema formulada pelo professor. Se acertar, pontos de vida serão subtraídos do "inimigo" e pontos resgatáveis atribuídos ao aluno; senão, pontos de vida serão subtraídos do aluno.

(6) O aluno poderá acessar as regras do jogo e definições de pontuações, padronizadas pelo sistema e personalizáveis pelo professor, assim como desempenho individual, de equipe e de turma.

(7) O professor poderá atribuir pontuação bônus e penalidades baseadas no comportamento do aluno (Tabela 2).

(8) A função chat estará disponível apenas para comunicação professor-aluno.

Table 1: Exemplos de feedback comportamental

\begin{tabular}{l|l}
\hline -XP & $+\mathbf{X P}$ \\
\hline - Escolher agir mal com os colegas & - Ajudar um colega em sala de aula \\
- Ser insubordinado & - Ser respeitoso durante apresentação \\
\hline
\end{tabular}

Quanto à frequência de reforços, a Tabela 2 identifica resumidamente os comportamentos adotados pelo usuário e os feedbacks em pontos de experiências (XP) e resgatáveis, adotados como pontos de batalha (PB). 


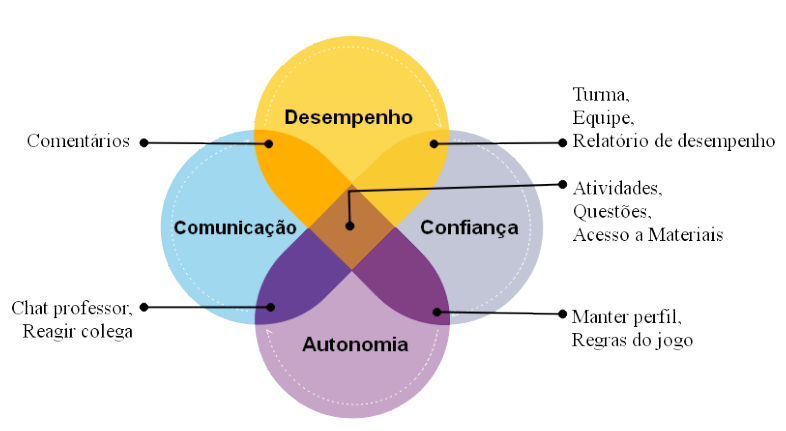

Figure 2: Relação entre "O que?" e "Quando?"

Table 2: Comportamentos e Recompensas

\begin{tabular}{l|c|c}
\hline Comportamento & XP & PB \\
\hline Realizar atividade da missão & $\mathrm{X}$ & $\mathrm{X}$ \\
\hline Acertar questão da Fase do Chefão & $\mathrm{X}$ & $\mathrm{X}$ \\
\hline Subir de Elo & - & $\mathrm{X}$ \\
\hline Reagir ao perfil do colega de turma & $\mathrm{X}$ & - \\
\hline Receber reação de perfil & $\mathrm{X}$ & - \\
\hline Comprar equipamentos & $\mathrm{X}$ & - \\
\hline Receber sentença positiva & $\mathrm{X}$ & - \\
\hline
\end{tabular}

Na dimensão “Como?", é definido o projeto da gamificação, como o detalhamento de dinâmicas e elementos de jogos mais adequados para cada situação (Tabela 3). Nessa dimensão, já com os objetivos motivacionais formalizados, também é importante definir os padrões de avaliação de desempenho de forma que sejam alcançáveis pelos aprendizes.

Na dimensão “Onde?” está o processo de desenvolvimento do sistema, podendo ser utilizadas técnicas da área de interface usuário máquina e engenharia de software. Além dos requisitos funcionais incorporados nas dimensões anteriores, são abordados outros requisitos do sistema.

Com uma identidade visual de estilo fantasy, a entidade Herói será mantida pela aquisição de equipamentos como: capacete, escudo, botas e armadura; herói e histórico de atribuições no sistema estarão visíveis em seu perfil. Quanto aos Elos de Equipe, a evolução se dará de acordo com acúmulo de Pontos de Experiência dos integrantes da equipe, com valores editáveis pelo professor (Tabela 4).

Table 4: Exemplo de relação entre elos e meta de XP definida como 1.000

\begin{tabular}{l|l|l|l|l|l|l}
\hline Elo de Equipe & Guerreiro & Elite & Mestre & Épico & Lenda & Mítico \\
\hline $\mathbf{X P}$ & 0 & 1.000 & 2.000 & 3.000 & 4.000 & 5.000 \\
\hline
\end{tabular}

O professor terá liberdade pra editar metas de pontuações, podendo definir a quantidade de: (1) XP para subir de nível do aluno; (2) XP para subir de elo de equipe; (3) XP atribuída ao dar/receber reação no perfil de colega de turma; (4) Pontos de batalha atribuída
Table 3: Componentes e elementos de jogo aplicados na jornada do jogador ("Quando?") em relação aos estímulos desejados ("Por Quê?")

\begin{tabular}{|c|c|c|c|c|c|}
\hline Quando? & Elementos de Jogo & Aprendizagem & Atenção & Satisfação & | Relevância \\
\hline $\begin{array}{l}\text { Manter per- } \\
\text { fil }\end{array}$ & $\begin{array}{l}\text { Avatar, bens virtu- } \\
\text { ais, personalização, } \\
\text { transações, desblo- } \\
\text { queio e coleções de } \\
\text { equipamentos, níveis, } \\
\text { pontos e feedback. }\end{array}$ & - & $\mathrm{X}$ & $\mathrm{X}$ & - \\
\hline $\begin{array}{l}\text { Acesso aos } \\
\text { materiais }\end{array}$ & $\begin{array}{l}\text { Desbloqueio de conteú- } \\
\text { dos, exploração, missão } \\
\text { e pontos. }\end{array}$ & $\mathrm{x}$ & $\mathrm{X}$ & $\mathrm{X}$ & $\mathrm{x}$ \\
\hline $\begin{array}{l}\text { Acesso } \\
\text { regras }\end{array}$ & $\begin{array}{l}\text { Loops de engajamento e } \\
\text { desafios. }\end{array}$ & - & - & - & $\mathrm{x}$ \\
\hline $\begin{array}{l}\text { Acompanhar } \\
\text { desem- } \\
\text { penho }\end{array}$ & $\begin{array}{l}\text { Cooperação, com- } \\
\text { petição e realizações. }\end{array}$ & $\mathrm{x}$ & $\mathrm{X}$ & $\mathrm{x}$ & - \\
\hline Comentários & $\begin{array}{l}\text { Feedback e relaciona- } \\
\text { mentos. }\end{array}$ & $\mathrm{x}$ & - & - & $\mathrm{X}$ \\
\hline $\begin{array}{l}\text { Chat pro- } \\
\text { fessor }\end{array}$ & $\begin{array}{l}\text { Feedback e relaciona- } \\
\text { mentos. }\end{array}$ & $\mathrm{x}$ & - & - & $\mathrm{X}$ \\
\hline $\begin{array}{l}\text { Reagir } \\
\text { colega de } \\
\text { turma }\end{array}$ & $\begin{array}{l}\text { Relacionamento, coop- } \\
\text { eração, recompensas e } \\
\text { pontos. }\end{array}$ & - & $\mathrm{X}$ & $\mathrm{x}$ & - \\
\hline Atividades & $\begin{array}{l}\text { Desbloqueio de conteú- } \\
\text { dos, exploração e mis- } \\
\text { são, pontos. }\end{array}$ & $\mathrm{X}$ & $\mathrm{X}$ & $\mathrm{X}$ & $\mathrm{X}$ \\
\hline Questões & $\begin{array}{l}\text { Combate, pontos, } \\
\text { placar, desafios, loops } \\
\text { de engajamento, re- } \\
\text { forço e feedback, } \\
\text { estados de vitória e } \\
\text { realizações. }\end{array}$ & $\mathrm{x}$ & $\mathrm{X}$ & $\mathrm{X}$ & $\mathrm{x}$ \\
\hline Equipes & $\begin{array}{l}\text { "Boss fights", relaciona- } \\
\text { mento, cooperação e } \\
\text { competição, recompen- } \\
\text { sas, pontos e criação de } \\
\text { ordem (níveis de Elos). }\end{array}$ & - & $\mathrm{X}$ & $\mathrm{X}$ & - \\
\hline Turma & $\begin{array}{l}\text { "Boss } \text { Fights", criação } \\
\text { de ordem, cooper- } \\
\text { ação, competição, } \\
\text { realizações e placar. }\end{array}$ & - & $\mathrm{X}$ & $\mathrm{X}$ & - \\
\hline
\end{tabular}

individualmente ao subir o elo de equipe; (5) XP atribuída ao comprar Equipamento.

Na dimensão “Quanto?”, são medidos os efeitos da gamificação, investigando o quanto a gamificação aplicada pôde estimular os comportamentos desejados no usuário, não abrangendo o sistema em sua totalidade. Em correspondência com o modelo ARCS, será utilizada a ferramenta Instructional Materials Motivational Scale (IMMS) para avaliar as reações dos usuários em ambientes autodirigidos [15]. Quanto à avaliação de aprendizagem, o indicador de desempenho pode ser mensurado pela taxa de acertos de atividades e por avaliações escritas, de acordo com a metodologia do professor.

\section{FERRAMENTA DE AVALIAÇÃO}

Em correspondência ao modelo ARCS e sua fundamentação teórica, duas ferramentas de medição foram projetadas para avaliar motivações em atividades e materiais [15]. Uma delas é a Instructional Materials Motivational Scale (IMMS), Pesquisa de Motivação de Materiais de Instrução em português, aplicada em contextos que utilizam materiais com instruções mediadas por computadores ou cursos on-line [15]. 
O IMMS incorpora abordagens teóricas em 36 questões com objetivo de mensurar o quão motivados os alunos estão em relação a um determinado curso, em contextos específicos. Além disso, suas questões podem ser adaptadas para se adequar às situações e à etapa do projeto avaliado, podendo ser usada como um pré-teste. A escala da resposta varia de 1 a 5 . Significa que em uma pesquisa, a pontuação mínima com 36 questões é de 36 e a máxima é de 180 , com um média de 108 [15]. Os valores variam porque nem todos os fatores abordados têm o mesmo número de questões.

No trabalho atual, o sistema em fase de protótipo (paper prototype) foi submetido a uma avaliação utilizando uma versão adaptada da ferramenta IMMS contendo 18 questões (Tabela 5), sendo aglutinados critérios essenciais de afirmações semelhantes do questionário original, subdivididas em cada fator do modelo ARCS

Table 5: Questões adaptadas da ferramenta IMMS

\begin{tabular}{|c|c|}
\hline \multirow[t]{7}{*}{ Atenção } & 1. O design do sistema é atraente \\
\hline & 2. Há algo interessante no sistema que chama atenção inicialmente \\
\hline & 3. A estrutura e elementos do sistema ajuda a manter a atenção \\
\hline & 4. O sistema estimula uma curiosidade sobre os conteúdos \\
\hline & 5. Pode deixar a mediação professor-aluno chata (reversa) \\
\hline & $\begin{array}{l}\text { 6. Tem muitos elementos no sistema o deixando cansativo e irri- } \\
\text { tante (reversa) }\end{array}$ \\
\hline & 7. O usuário pode encontrar surpresas agradáveis \\
\hline \multirow[t]{4}{*}{ Relevância } & 8. O sistema tem elementos conhecidos pelos futuros usuários \\
\hline & 9. Pode ser utilizado de acordo com as formas de aprendizado \\
\hline & 10. Concluir tarefas se mostra como um importante sucesso \\
\hline & $\begin{array}{l}\text { 11. Possui ferramentas e elementos relevantes para o professor no } \\
\text { gerenciamento de desempenho }\end{array}$ \\
\hline \multirow[t]{3}{*}{ Confiança } & 12. O sistema é fácil de se entender \\
\hline & 13. Deixa claro o que deve ser feito \\
\hline & 14. Possibilita uma auto-confiança na aprendizagem \\
\hline \multirow[t]{4}{*}{ Satisfação } & 15. Possibilita o sentimento de conquista e realização \\
\hline & $\begin{array}{l}\text { 16. Feedbacks suficientes para manter o sentimento de que "valeu } \\
\text { a pena" }\end{array}$ \\
\hline & 17. Recompensas justas \\
\hline & $\begin{array}{l}\text { 18. O projeto possibilita o prazer no processo de mediação da } \\
\text { aprendizagem }\end{array}$ \\
\hline
\end{tabular}

Foi utilizada uma escala de pontuação de cinco valores que variam de acordo com o nível de concordância do avaliador com cada afirmação da questão: (1) Discordo Totalmente; (2) Discordo; (3) Indiferente (neutro); (4) Concordo; (5) Concordo Totalmente.

\section{DISCUSSÕES E ANÁLISES PRELIMINARES}

Para avaliar os possíveis efeitos da gamificação em relação aos objetivos das dimensões, a avaliação do paper prototype foi aplicada com 11 (onze) professores mestres e doutores das áreas de Sistemas de Informação, Engenharia de Software, Ciências da Computação, Análise de Sistemas e Recuperação da Informação, atuantes nos níveis de ensino superior, técnico e cursos livres, em instituições públicas e privadas. A opção por usar paper prototype se deu pela possibilidade de uma implementação real criar ruídos, uma vez que envolve outras decisões de escolhas tecnológicas e design que podem influenciar nos resultados [25].

As pontuações das respostas obtidas de cada avaliador foram separadas por fatores e somadas por questão; as questões fraseadas de forma negativa foram convertida em uma escala reversa para facilitar a análise. As pontuações médias de cada fator $(p)$ foram calculadas pela Equação 3 onde, $i$ é quantidade de avaliadores, $j$ é quantidade de questões do fator e $R_{m}$ é resposta da $m$-ésima avaliação.

$$
p=\frac{\sum_{n=1}^{i} \sum_{m=1}^{j} R_{m}}{i}
$$

Com base nas médias e nas questões de cada fator ARCS, foi adotado um indicador de aproveitamento (AP) para análise e avaliação [21]. O indicador é a normalização de $p$ (média) para uma escala percentual demonstrado na Equaçõ 4, considerando um AP em 100\% nos casos em que os avaliadores atribuíram notas máximas.

$$
A P=\frac{(\text { media }-M I N)}{(M A X-M I N) / 100}
$$

\section{Table 6: Análise ARCS paper prototype}

\begin{tabular}{l|c|c|c|c}
\hline & A & R & C & S \\
\hline Mínimo & 7 & 4 & 3 & 4 \\
\hline Máximo & 35 & 20 & 15 & 20 \\
\hline Média (p) & 32,09 & 19,27 & 14,09 & 19,00 \\
\hline AP & $89,61 \%$ & $95,45 \%$ & $92,42 \%$ & $93,75 \%$ \\
\hline
\end{tabular}

Os valores de AP, ilustrados na Tabela 6, nos permitem visualizar os fatores atendidos, apresentando a motivação apontada pelos avaliadores, variando entre $89,61 \%$ e $93,75 \%$. A seguir são apresentadas as frequências relativas das respostas de acordo com as afirmações do questionário e pontuações.

\subsection{Atenção}

Na Figura 3, é possível visualizar o grau de concordância que os elementos e o design do sistema despertam e ajudam a manter a atenção do usuário no ambiente, mesmo em sua fase de protótipo.

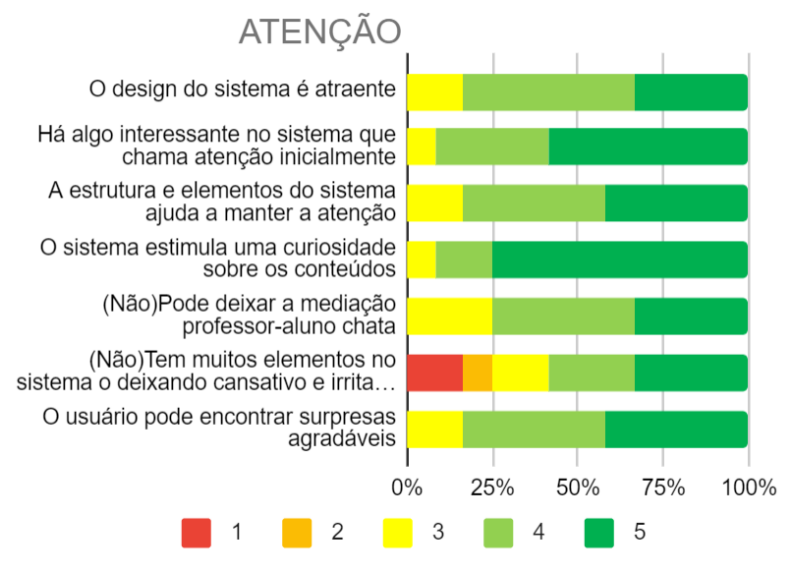

Figure 3: Reação do usuário na categoria Atenção.

Na afirmação "(Não) Tem muitos elementos no sistema o deixando cansativo e irritante", é possível ver a variação de pontuação que influenciou na pequena queda do indicador AP em relação aos demais fatores $(\mathrm{AP}=89,61 \%)$. A indiferença pode ser justificada 
pelas diferenças de gerações entre os professores e entre a relação professor-aluno, contrastando possíveis desafios e rupturas na percepção de mundo em uma realidade tecnológica envolvendo sistemas e games [10]. A Figura 4 exibe a tela de perfil do aluno no sistema em protótipo.

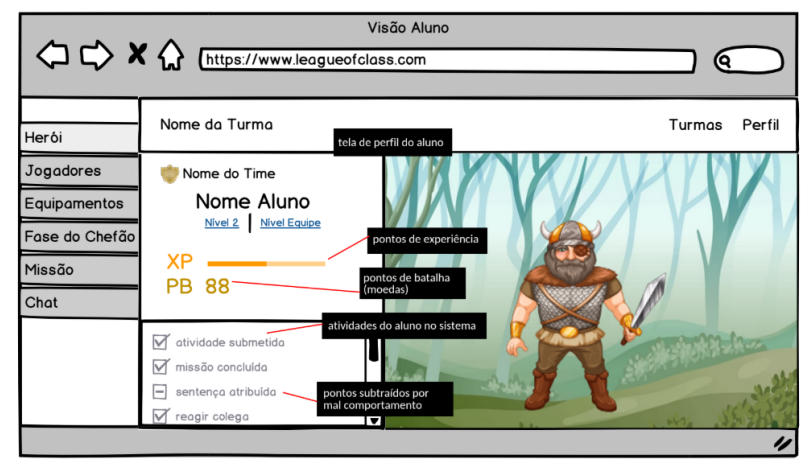

Figure 4: Imagens do mockup do sistema: Perfil do Aluno

\subsection{Relevância}

No fator Relevância o indicador AP obteve o maior valor, 95,45\% (coluna R da Tabela 6), como apresentado na Figura 5.

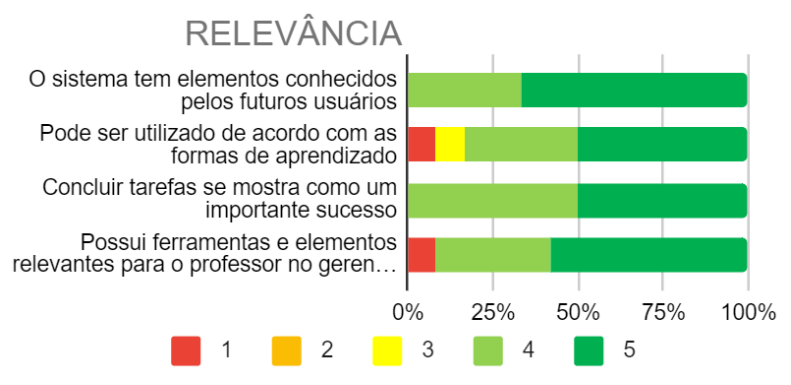

Figure 5: Reação do usuário na categoria Relevância.

Isso indica que o sistema se mostrou promissor quanto ao seu papel de auxílio ao professor, abrindo a possibilidade de ampliar metodologias adotadas e abranger variadas formas de aprendizado. Contudo, é necessária uma avaliação mais detalhada com estudantes como usuários finais. A Figura 6 exibe a tela do mapa da missão representada no protótipo do sistema.

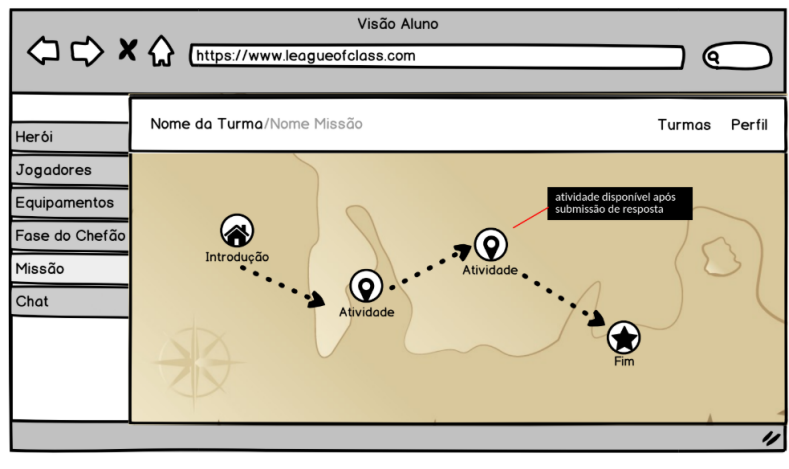

Figure 6: Imagens do mockup do sistema: Mapa de atividades

\subsection{Confiança}

Na Figura 7 é possível observar que $100 \%$ dos participantes tiveram a percepção de que a estrutura e elementos do sistema são fáceis se de entender. O fator obteve o indicador AP de $92,42 \%$ (coluna $C$ da Figura 6), sugerindo que o sistema possibilita um objetivo de aprendizagem claro e bem estruturado, de acordo com o planejamento e aplicação do professor, favorecendo um possível sentimento de controle pessoal do aluno. Esse princípio é uma das áreas de pesquisas motivacionais mais populares, pois está diretamente relacionada à produção de resultados e regulação de esforços para uma tarefa bem sucedida [16].

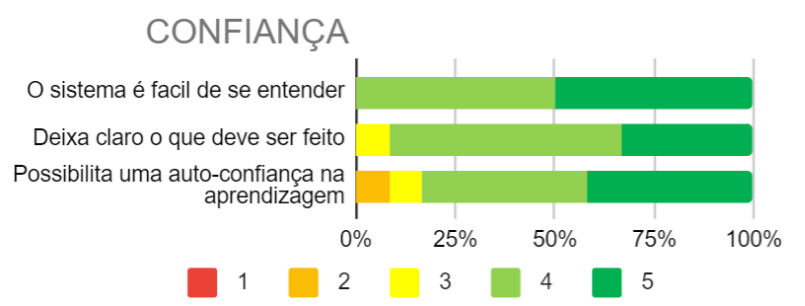

Figure 7: Reação do usuário na categoria Confiança.

A Figura 8 exibe a tela em que o aluno responde o desafio da Fase do Chefão, apresentada no protótipo.

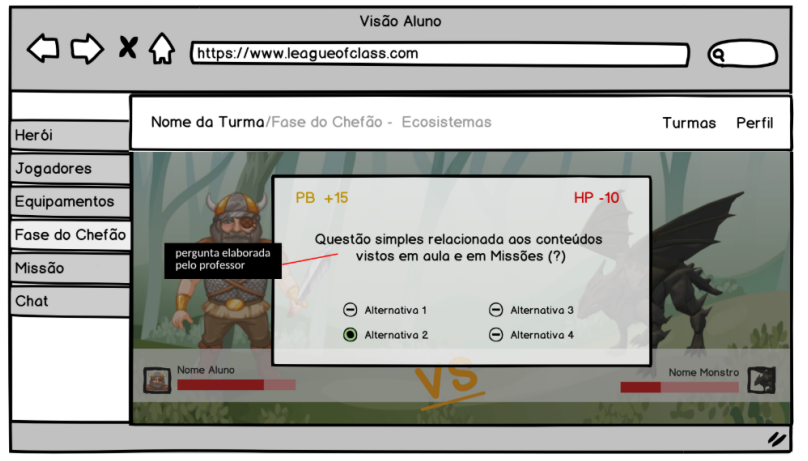

Figure 8: Imagens do mockup do sistema: Tela Fase do Chefão 


\subsection{Satisfação}

Referente ao fator Satisfação, o gráfico da Figura 9 confirma o alto grau de satisfação percebida no sistema ( $\mathrm{AP}=93,75 \%)$, possuindo feedbacks como elementos chave de fonte de informação ao aluno sobre seu desempenho, no objetivo de ementar comportamentos e pensamentos positivos sobre sua experiência [16].

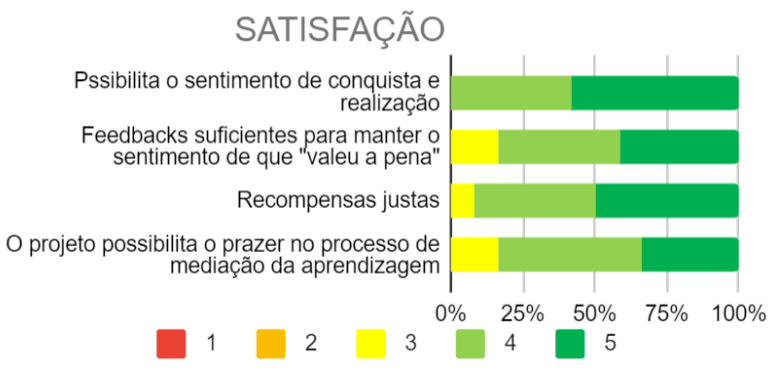

Figure 9: Reação do usuário na categoria Satisfação.

\subsection{Questões abertas discursivas}

Em uma questão discursiva opcional de título "Quais aspectos deste sistema foram mais úteis ou valiosos?", a avaliação recebeu respostas de professores como "o papel que o trabalho em equipe tem dentro do sistema", "interessante a construção do personagem", "os gráficos de avaliação e a consolidação dos dados", "missões e os mapas são excelentes ferramentas de acompanhamento" e "forma como os elementos de Gamificação explorados [...]. Adorei a ideia do professor poder cadastrar as questões [...]. Esse seria um sistema que com absoluta certeza eu iria utilizar!”. Os professores sugeriram, em trabalhos futuros, que o sistema explorasse mais recursos visuais como ícones de menu e cores no sistema, que pudesse ter outros temas e personagens da cultura pop, assim como desafios entre colegas de turma.

Foi perguntado, também, "Em um contexto geral, como a metodologia de gamificação poderia auxiliar no ensino da computação?”.

Respostas como: "A computação é muito rica tanto em assuntos teóricos quanto práticos, neste contexto, muitos assuntos vistos em classe servem de base para outras temáticas mais aprofundadas. A Gamificação, neste sentido, ajudaria no processo de ensino-aprendizagem de forma que a cada fase vencida (ou concluída), o aluno iria se sentir mais apto ou confiante de partir para o próximo assunto (ou nível) da disciplina. A pontuação ou até mesmo a evolução do aluno em um sistema gamificado ajudam muito no que diz respeito à autoconfiança e motivação do aluno, visto que temos muitos casos de desistência dos alunos em disciplinas muito aplicadas e/ou complexas."

As respostas dão indícios de que os professores acreditam que a gamificação, nesse contexto, é uma forma de obter uma atração contextual ("a fixação da atenção ao assunto lecionado") e motivar intrinsecamente os estudantes no processo de aprendizagem pela competição e a irem em busca de conhecimento, estimulados pelos desafios, investindo mais tempo em suas atividades. "Para muitos alunos a principal recompensa é passar na disciplina com boa nota", podendo ser interessante relacionar as recompensas com as notas da disciplina.

\section{CONSIDERAÇÕES FINAIS}

Este trabalho apresentou a proposta de gamificação estrutural League of Class como um sistema gerenciador de pontuações para apoio ao professor no encaminhamento de ações, definindo uma abordagem relacionada à motivação para o aprendizado na perspectiva do modelo ARCS e com seus elementos planejados por meio do framework $5 \mathrm{~W} 2 \mathrm{H}$. O sistema foi avaliado por onze professores de áreas da computação em níveis de ensino superior, técnico e cursos livres, através da adaptação da ferramenta Instructional Materials Motivational Scale (IMMS). Com base nas respostas, é possível considerar que a estrutura do sistema ajuda a manter a atenção e estimular a curiosidade. Os avaliadores concordaram que o sistema possui elementos relevantes para o professor na gerência de atividades e também para estimular o engajamento do aluno nas submissões de respostas, abrindo a possibilidade da construção da auto-confiança na aprendizagem e o prazer no processo de mediação da aprendizagem.

Uma das limitações do trabalho é a falta da aplicação do ambiente em um contexto real de aprendizagem. Dentre os avaliadores, $84,2 \%$ (dezesseis) acreditam que a utilização do sistema apresentado em um contexto educacional poderia influenciar positivamente em notas e índices de desempenho, e 15,8\% (três) apresentaram um "talvez" como resposta à afirmação. 89,5\% (dezessete) dos avaliadores afirmaram que utilizariam o sistema em suas aulas como ferramenta complementar, sem considerar as dificuldades tecnológicas de seu contexto real de atuação. Atualmente, o sistema está em fase de implementação e, como trabalhos futuros, os autores pretendem investigar mais a fundo a dimensão “Quanto?”, com a análise da aplicação do sistema em contextos reais de aprendizagem, e contribuir para a discussão sobre efeitos da gamificação na educação.

\section{REFERENCES}

[1] Flora Alves. 2015. Gamification: Como criar experiências de aprendizagem engajadoras. DVS editora.

[2] Weverson Ribeiro Alves et al. 2018. Uma Proposta de Ensino de Matemática: O Uso do Game Dota 2 como Ferramenta Pedagógica Matemática. (2018).

[3] Inês Araújo and Ana Amélia Carvalho. 2017. Capacitar professores para o uso da gamificação. Atas do XIX Simpósio Internacional de Informática Educativa e VIII Encontro do CIED-III Encontro Internacional (2017), 264-269.

[4] Rafaela Caetano. 2019. ESPN. A gente explica e você vira fã: $O$ que são os mobas? http://www.espn.com.br/infografico/a-gente-explica-e-voce-vira-fa-oque-sao-os-mobas/

[5] Gustavo Chiminazzo and Victor Marques. 2020. ISTOÉ DINHEIRO: E-sports não é (mais) brincadeira. https://www.istoedinheiro.com.br/e-sports-nao-e-maisbrincadeira/

[6] Gabriel Conejo, Isabela Gasparini, and Marcelo da Silva Hounsell. 2019. Detalhando a Motivação em um Processo de Gamificação. In Anais dos Workshops do Congresso Brasileiro de Informática na Educação, Vol. 8. 1114.

[7] Andreza Regina Lopes da Silva, Araci Hack Catapan, Cláudio Henrique da Silva, Eliseo Berni Reategui, Fernando José Spanhol, Ildo Francisco Golfetto, Juliana Bordinhão Diana, Lynn Rosalina Gama Alves, Luciane Maria Fadel, Luís H Lindner, et al. 2014. Gamificação na educação. Pimenta Cultural.

[8] Merhi Daychoum. 2018. 40+ 20 ferramentas e técnicas de gerenciamento. Brasport.

[9] Christo Dichev and Darina Dicheva. 2017. Gamifying education: what is known, what is believed and what remains uncertain: a critical review. International journal of educational technology in higher education 14, 1 (2017), 9.

[10] Elydio dos Santos Neto and Edgar Silveira Franco. 2010. Os professores e os desafios pedagógicos diante das novas gerações: considerações sobre o presente e o futuro. Revista de Educação do COGEIME 19, 36 (2010), 9-25.

[11] Amanda Haiduk and Jean Carlos Hennrichs. 2018. CALL OF CODE: UMA PLATAFORMA DE ENSINO INTERDISCIPLINAR À LÓGICA DE PROGRAMAÇÃO, AMBIENTADA NA SEGUNDA GUERRA MUNDIAL. Seminário de Iniciação Científica, Seminário Integrado de Ensino, Pesquisa e Extensão e Mostra Universitária (2018). 
[12] Jean Carlos Hennrichs and Amanda Haiduk. 2020. CALL OF CODE: UMA PLATAFORMA DE ENSINO DE LÓGICA DE PROGRAMAÇÃO, INTERDISCIPLINAR E AMBIENTADA NA SEGUNDA GUERRA MUNDIAL. Seminário de Iniciação Científica e Seminário Integrado de Ensino, Pesquisa e Extensão (2020), e25893-e25893.

[13] Karl M Kapp. 2012. The gamification of learning and instruction: game-based methods and strategies for training and education. John Wiley \& Sons.

[14] Karl M Kapp, L Blair, and R Mesch. 2014. The gamification of learning and instruction: Ideas into Practice.

[15] John M Keller. 2009. Motivational design for learning and performance: The ARCS model approach. Springer Science \& Business Media.

[16] John M Keller. 2010. Five fundamental requirements for motivation and volition in technology-assisted distributed learning environments. Revista Inter Ação 35 2 (2010), 305-322.

[17] Ana Carolina Tomé Klock, Isabela Gasparini, and Marcelo Soares Pimenta. 2016. 5W2H Framework: a guide to design, develop and evaluate the user-centered gamification. In Proceedings of the 15th Brazilian Symposium on Human Factors in Computing Systems. 1-10.

[18] Rosilene Agapito Llarena, Emeide Nóbrega Duarte, and Suzana De Lucena Lira. 2016. A Arquitetura Da Informação à luz da teoria de Piaget: uma possibilidade epistemológica para a gestão do conhecimento. Perspectivas em Gestão \& Conhecimento 6, 1 (2016), 36-52.

[19] A Marczewski. 2013. A player type framework for gamification design. URL https://www.gamified.uk/user-types/ (2013).

[20] Andrzej Marczewski. 2015. Even Ninja Monkeys like to play. CreateSpace Indep. Publish Platform, Charleston, Chapter User Types (2015), 69-84.
[21] José Arnaldo Barra Montevechi, Fabiano Leal, Rafael de Carvalho Miranda, and Tábata Fernandes Pereira. 2013. Interactive learning of modeling and discreteevents simulation through Lego ${ }^{\circledR}$ parts. In 2013 Winter Simulations Conference (WSC). IEEE, 3556-3567.

[22] Alberto Mora, Daniel Riera, Carina González, and Joan Arnedo-Moreno. 2017. Gamification: a systematic review of design frameworks. fournal of Computing in Higher Education 29, 3 (2017), 516-548.

[23] Marçal Mora-Cantallops and Miguel-Ángel Sicilia. 2018. MOBA games: A literature review. Entertainment computing 26 (2018), 128-138.

[24] Andre Nunes, Isabela Gasparini, and Marcelo da Silva Hounsell. [n.d.]. Gamificação de um sistema de Acompanhamento Diário de Dependentes Químicos. ([n.d.]).

[25] Rita Orji, Gustavo F Tondello, and Lennart E Nacke. 2018. Personalizing persuasive strategies in gameful systems to gamification user types. In Proceedings of the 2018 CHI Conference on Human Factors in Computing Systems. 1-14.

[26] Marcela Pessoa, David Fernandes, Leandro Silva Galvão de Carvalho, Elaine Oliveira, Walter Nakamura, and Tayana Conte. 2019. CodePlay: Uma Plataforma de Gamificação baseada em Jogos de RPG Multiplayer. In Brazilian Symposium on Computers in Education (Simpósio Brasileiro de Informática na Educação-SBIE), Vol. 30. 843.

[27] Armando M Toda, Wilk Oliveira dos Santos, Ana CT Klock, Isabela Gasparini, Ig Ibert Bittencourt, and Seiji Isotani. 2018. Frameworks para o planejamento da gamificaçao em contextos educacionais-uma revisao da literatura nacional. RENOTE-Revista Novas Tecnologias na Educação 16, 2 (2018), 505-514.

[28] Kevin Werbach and Dan Hunter. 2012. For the win: How game thinking can revolutionize your business. Wharton Digital Press. 